\title{
L-FUZZIFYING TOPOLOGICAL VECTOR SPACES
}

\author{
CONG-HUA YAN AND CONG-XIN WU
}

Received 5 January 2005 and in revised form 28 May 2005

The main purpose of this paper is to introduce a concept of $L$-fuzzifying topological vector spaces (here $L$ is a completely distributive lattice) and study some of their basic properties. Also, a characterization of such spaces in terms of the corresponding $L$-fuzzifying neighborhood structure of the zero element is given. Finally, the conclusion that the category of $L$-fuzzifying topological vector spaces is topological over the category of vector spaces is proved.

\section{Introduction and preliminaries}

The theory of topological vector spaces is an important branch of modern analysis. There are abroad applications in mathematics and other subjects. So it is natural to consider the reasonable generalization of the topological vector spaces in the setting of many valued sets. In 1977, Katsaras and Liu combined the structure of fuzzy topology with the structure of vector spaces in [6] for the first time and introduced the concept of fuzzy topological vector spaces. In 1997, Fang and Yan in [2] generalized it to the concept of $L$-topological vector spaces (where $L$ is a Hutton algebra). From then on, many properties of $L$-topological vector spaces were discussed by Yan and Fang. On the other hand, Höhle [4] introduced in 1980 the concept of fuzzy measurable spaces with the idea of giving degrees in $[0,1]$ to some topological terms rather than 0 or 1 . Sharing similar ideas to those above, Ying in [9] gave a new approach for fuzzy topology from a logical point of view, that is, a concept of fuzzifying topology was given. Recently, the relations between fuzzifying topological spaces and $L$-topological spaces have received wide attention [7, 12]. Specially Liu and Zhang proved the category of $L$-fuzzifying topological spaces is concretely isomorphic to the category of Lowen spaces in [7]. Moreover, Höhle [5] and Zhang and Xu [12, 13] introduced the concept of topological Lfuzzifying neighborhood structures, respectively. The equivalences between $L$-fuzzifying topologies and topological $L$-fuzzifying neighborhood structures have also been independently established by them. In this paper, we will combine the structure of $L$-fuzzifying topologies with the structure of vector spaces and introduce the concept of $L$-fuzzifying topological vector spaces and some basic properties of these spaces are studied. Then 
we obtain a characterization of $L$-fuzzifying topological vector spaces in terms of the $L$ fuzzifying neighborhood structure of the zero element. We will prove that the category of $L$-fuzzifying topological vector spaces $L$-FYTVS is topological over the category of vector spaces VEC.

First we fix some notations throughout this paper. $L$ denotes a completely distributive lattice if not otherwise stated. 0 and 1 are its top element and bottom element, respectively. $M(L)$ denotes the set of all nonbottom coprimes $L$. $\mathbb{K}$ is a nondiscrete-valued field. For $a, b \in L$, we say $a$ is wedge below $b$, in symbols, $a \triangleleft b$, if for every arbitrary subset $D \subseteq L, \vee D \geq b$ implies that $a \leq d$ for some $d \in D$. For every $x \in X, \dot{x}$ denotes the principal filter generated by $x$, and for all $x \in U \subset X, \dot{x} \mid U$ denotes the collection $\{V \subseteq U \mid x \in V\}$.

Definition 1.1 (Höhle [5], Ying [9], Zhang [12]). An L-fuzzifying topology on the set $X$ is a function $\tau: 2^{X} \rightarrow L$ which fulfills, for all $U, V, U_{j} \subset X(j \in J)$, the following:

(FY1) $\tau(\varnothing)=\tau(X)=1$;

(FY2) $\tau(U \cap V) \geq \tau(U) \wedge \tau(V)$;

(FY3) $\tau\left(\bigcup_{j \in J} U_{j}\right) \geq \wedge_{j \in J} \tau\left(U_{j}\right)$.

If $\tau$ is an $L$-fuzzifying topology on $X$, then $(X, \tau)$ is an $L$-fuzzifying topological space.

The value $\tau(U)$ is interpreted as the degree of openness of $U$. A continuous function between $L$-fuzzifying topological spaces is a function $f:(X, \tau) \rightarrow(Y, \eta)$ such that $\tau\left(f^{-1}(U)\right) \geq \eta(U)$ for all $U \in 2^{Y}$.

Definition 1.2 (Höhle [5], Liu and Zhang [7, 12]). An L-fuzzifying neighborhood structure on a set $X$ is a family of functions $P=\left\{p_{x}: 2^{X} \rightarrow L \mid x \in X\right\}$ with the following conditions, for all $x \in X, U, V \subset X$ :

(LN1) $p_{x}(X)=1$

(LN2) $p_{x}(U)>0 \Rightarrow x \in U$;

(LN3) $p_{x}(U \cap V)=p_{x}(U) \wedge p_{x}(V)$.

The pair $(X, P)$ is called an $L$-fuzzifying neighborhood space, and it will be called topological if it satisfies moreover the following condition, for all $x \in X, U \subset X$ :

(LN4) $p_{x}(U)=\bigvee_{V \in \dot{x} \mid U} \bigwedge_{y \in V} p_{y}(V)$.

A continuous function between $L$-fuzzifying neighborhood spaces $(X, P)$ and $(Y, Q)$ is a map $f: X \rightarrow Y$ such that for all $x \in X, U \subset Y, p_{x}\left(f^{-1}(U)\right) \geq q_{f(x)}(U)$.

Suppose $\tau: 2^{X} \rightarrow L$ is an $L$-fuzzifying topology, for all $x \in X, U \subset X$ and let $p_{x}^{\tau}(U)=$ $\bigvee_{V \in \dot{x} \mid U} \tau(V)$; then we have the following lemma.

Lemma 1.3 (Zhang [12]). (1) $\left(X, P^{\tau}\right)$ is a topological L-fuzzifying neighborhood space, where $P^{\tau}=\left\{p_{x}^{\tau} \mid x \in X\right\}$.

(2) For all $U \subset X, \tau(U)=\bigwedge_{x \in U} p_{x}^{\tau}(U)$.

(3) If $f:(X, \tau) \rightarrow(Y, \eta)$ is continuous, then $f:\left(X, P^{\tau}\right) \rightarrow\left(Y, Q^{\eta}\right)$ is continuous.

Conversely, given a topological $L$-fuzzifying neighborhood space $(X, P)$, for all $U \subset X$, letting $\tau_{P}(U)=\bigwedge_{x \in U} p_{x}(U)$, the following conclusions hold.

Lemma 1.4 (Zhang [12]). (1) $\tau_{P}$ is an L-fuzzifying topology on $X$.

(2) For all $x \in X, U \subset X, p_{x}(U)=\bigvee_{V \in \dot{x} \mid U} \tau_{P}(V)$. 
(3) If $f:(X, P) \rightarrow(Y, Q)$ is a continuous function, then $f:\left(X, \tau_{P}\right) \rightarrow\left(Y, \eta_{Q}\right)$ is continuous.

By [5, Proposition 3.13 and Proposition 3.14], the next lemma is easy to obtain.

Lemma 1.5 (Höhle [5]). Let $X \neq \varnothing$ be a set, a family of functions $P=\left\{p_{x}: 2^{X} \rightarrow L \mid x \in X\right\}$ satisfying conditions (LN1), (LN2), and (LN3). Then the following conditions are equivalent.

(LN4) $p_{x}(U)=\bigvee_{V \in \dot{x} \mid U} \bigwedge_{y \in V} p_{y}(V)$.

(u4) $p_{x}(U) \leq \bigvee_{y \notin B}\left[p_{y}(U) \vee p_{x}(B)\right]$, for all $B \subseteq X$.

From [10, Definition 3.1], if $\tau_{1}$ and $\tau_{2}$ are two $L$-fuzzifying topologies on $X_{1}$ and $X_{2}$, respectively, then a subbase $\varphi$ of the $L$-fuzzifying product topology $\tau_{1} \times \tau_{2}$ may be defined as follows: $\varphi\left(p_{i}^{-1}\left(U_{i}\right)\right)=\tau_{i}\left(U_{i}\right)$ for all $U_{i} \in X_{i}, i=1,2$; otherwise its value is 0 . At the same time, we easily find that the $L$-fuzzifying neighborhood structure $\left(p_{x}\right)_{x \in X}$ corresponding to the $L$-fuzzifying product topology may be described as follows:

$$
p_{(x, y)}(A)=\bigvee_{U \times V \subseteq A} p_{x}(U) \bigwedge p_{y}(V), \quad A \subseteq X_{1} \times X_{2}
$$

Lemma 1.6 (Gierz et al. [3]). Let L be a completely distributive lattice; then the following relations hold:

(1) for each $a \in L, a=\vee\{b \in M(L) \mid b \triangleleft a\}$;

(2) for $a \in L$ and $D \subset L$, if $a \triangleleft \bigvee D$, then there exists $d \in D$ such that $a \triangleleft d$;

(3) for all $a, b \in L, a \triangleleft b$ implies (there exists $c \in M(L)$ ) that $a \triangleleft c \triangleleft b$; furthermore, $a \neq b$ and $a \triangleleft b$ together imply (there exists $c \in M(L), a \neq c$ ) that $a \triangleleft c \triangleleft b$.

In fact, Lemma 1.6(1) is exactly the one on [3, Section 2.29, Exercise (iii), page 204], and Lemma 1.6(3) is the one on [3, Section 2.29, Exercise (iv), page 204]. In particular, Lemma 1.6(2) is a corollary of Lemma 1.6(1), (3).

\section{2. $L$-fuzzifying topological vector spaces}

Definition 2.1. Let $X$ be a vector space over $\mathbb{K}$ and $\tau$ an $L$-fuzzifying topology on $X$. The pair $(X, \tau)$ is said to be an $L$-fuzzifying topological vector space, if the following two mappings are continuous:

(1) $f: X \times X \rightarrow X,(x, y) \mapsto x+y$;

(2) $g: \mathbb{K} \times X \rightarrow X,(k, x) \mapsto k x$,

where $X \times X$ and $\mathbb{K} \times X$ are equipped with the corresponding $L$-fuzzifying product topologies $\tau \times \tau$ and $\mathfrak{J}_{\mathbb{K}} \times \tau$ (here $\mathfrak{J}_{\mathbb{K}}$ is an $L$-fuzzifying topology determined by the crisp neighborhood structure on $\mathbb{K})$, respectively.

Remark 2.2. A usual topological vector space can be regarded as an $L$-fuzzifying topological vector space (with the $L$-fuzzifying topology determined by the crisp neighborhood structure). When $L=[0,1]$, our definition of $L$-fuzzifying topological vector spaces is different from that in [11]. In [11], the author gave the definition with the fuzzifying neighborhood structure directly. Our definition begins with the continuity of the operations on the vector structure. From [11, Definition 3] and Proposition 2.3 in our paper, 
it is easy to verify that the two definitions are equivalent with $L=[0,1]$. In other words, our definition is a generalization of that in [11].

Proposition 2.3. The mapping $f$ in Definition 2.1 is continuous if and only if condition (LFN1) holds, that is, for every $x, y \in X, W \subseteq X, \bigvee_{U+V \subseteq W} p_{x}(U) \wedge p_{y}(V) \geq p_{x+y}(W)$ (here $P=\left\{p_{x} \mid x \in X\right\}$ is an L-fuzzifying neighborhood structure determined by $\tau$ ).

Proof. Necessity. Since $f:(X, \tau) \times(X, \tau) \rightarrow(X, \tau)$ is continuous, it follows from Lemma 1.3 that the mapping $f:(X, P) \times(X, P) \rightarrow(X, P)$ is continuous. Thus for every $x, y \in$ $X, W \subseteq X$, we have $p_{(x, y)}\left(f^{-1}(W)\right) \geq p_{x+y}(W)$. On the other hand, $f^{-1}(W)=\bigcup_{j \in J}\left\{U_{j} \times\right.$ $\left.V_{j} \mid U_{j}+V_{j} \subseteq W\right\}$. So we have

$$
\bigvee_{U+V \subseteq W} p_{x}(U) \bigwedge p_{y}(V)=p_{(x, y)}\left(f^{-1}(W)\right) \geq p_{x+y}(W)
$$

This means that the necessity is proved.

Sufficiency. From the above proof, for each $x, y \in X, W \subseteq X$, we have

$$
p_{(x, y)}\left(f^{-1}(W)\right)=\bigvee_{U+V \subseteq W} p_{x}(U) \bigwedge p_{y}(V) \geq p_{x+y}(W)
$$

So the mapping $f:(X, P) \times(X, P) \rightarrow(X, P)$ is continuous; it follows from Lemma 1.4 that the mapping $f:(X, \tau) \times(X, \tau) \rightarrow(X, \tau)$ is continuous. This completes the proof.

Proposition 2.4. The mapping $g$ in Definition 2.1 is continuous if and only if condition (LFN2) holds, that is, for every $k_{0} \in \mathbb{K}, x \in X, W \subseteq X, \underset{\substack{M U \subseteq W \\ k_{0} \in M}}{\operatorname{Pa}} p_{x}(U) \geq p_{k_{0} x}(W)$ (here $P=\left\{p_{x} \mid x \in X\right\}$ is an L-fuzzifying neighborhood structure determined by $\tau$ and $M=\{k \mid$ $\left.\left.\exists \varepsilon>0,\left|k-k_{0}\right|<\varepsilon\right\}\right)$.

The proof of Proposition 2.4 is similar to that of Proposition 2.3, so we leave it to readers.

Propositions 2.3 and 2.4 give the conditions of the mappings $f$ and $g$ in Definition 2.1 with the help of the $L$-fuzzifying neighborhood structure. Moreover, we may obtain the following equivalent conditions by $L$-fuzzifying topologies directly.

Proposition 2.5. (1) The mapping $f$ in Definition 2.1 is continuous if and only if condition (LFN1)' holds, that is, for every $x, y \in X, A \subseteq X$ with $x+y \in A$,

$$
\bigvee_{\substack{B+C \subset A \\ B \in \dot{x}, C \in \dot{y}}} \tau(B) \bigwedge \tau(C) \geq \tau(A)
$$

(2) The mapping $g$ in Definition 2.1 is continuous if and only if condition (LFN2)' holds, that is, for every $k_{0} \in \mathbb{K}, x \in X, A \subseteq X$ with $k_{0} x \in A, \bigvee_{\substack { M B \dot{x}, k_{0} \in M \\ \begin{subarray}{c}{B \\ \text { a }{ M B \dot { x } , k _ { 0 } \in M \\ \begin{subarray} { c } { B \\ \text { a } } }\end{subarray}} \tau(B) \geq \tau(A)$. The meaning of $M$ is the same as in Proposition 2.4.

Proof. (1) It is sufficient to prove that condition (LFN1) is equivalent to condition $(\mathrm{LFN} 1)^{\prime}$. 
In fact, if (LFN1) is satisfied, then for every $x, y \in X, A \subseteq X$ with $x+y \in A$, we have

$$
\begin{aligned}
\tau(A) & =\bigwedge_{x \in A} p_{x}(A) \leq p_{x+y}(A) \leq \bigvee_{B+C \subseteq A} p_{x}(B) \bigwedge p_{y}(C) \\
& =\bigvee_{B+C \subseteq A}\left(\bigvee_{x \in B_{1} \subseteq B} \tau\left(B_{1}\right) \bigwedge \bigvee_{y \in C_{1} \subseteq C} \tau\left(C_{1}\right)\right) \\
& =\bigvee_{B+C \subseteq A} \bigvee_{\substack{B_{1} \in \dot{x}\left|B \\
C_{1} \in \dot{y}\right| C}} \tau\left(B_{1}\right) \bigwedge \tau\left(C_{1}\right)=\bigvee_{\substack{B+C \subseteq A \\
x \in B, y \in C}} \tau(B) \bigwedge \tau(C) .
\end{aligned}
$$

Conversely, suppose that (LFN1)' is satisfied. Then for all $x, y \in X, W \subseteq X$, we have

$$
\begin{aligned}
p_{x+y}(W) & =\bigvee_{x+y \in W_{1} \subseteq W} \tau\left(W_{1}\right) \leq \bigvee_{\substack{U+V \subseteq W_{1} \subseteq W \\
x \in U, y \in V}} \tau(U) \bigwedge \tau(V) \\
& \leq \bigvee_{U+V \subseteq W} p_{x}(U) \bigwedge p_{y}(V) .
\end{aligned}
$$

(2) It suffices to prove that condition (LFN2) is equivalent to (LFN2)'.

If condition (LFN2) is satisfied, then for every $k_{0} \in \mathbb{K}, x \in X, A \subseteq X$ with $k_{0} x \in A$, we have

$$
\begin{aligned}
\tau(A) & =\bigwedge_{y \in A} p_{y}(A) \leq p_{k_{0} x}(A) \leq \bigvee_{\substack{M U \subseteq A \\
k_{0} \in M}} p_{x}(U) \\
& =\bigvee_{\substack{M U \subseteq A \\
k_{0} \in M}} \bigvee_{B \in \dot{x} \mid U} \tau(B) \leq \bigvee_{\substack{M B \subseteq A \\
x \in B, k_{0} \in M}} \tau(B) .
\end{aligned}
$$

On the contrary, if condition (LFN2)' is satisfied, then for all $k_{0} \in \mathbb{K}, x \in X, W \subseteq X$, we have

$$
\begin{aligned}
p_{k_{0} x}(W) & =\bigvee_{k_{0} x \in A_{1} \subseteq W} \tau\left(A_{1}\right) \leq \bigvee_{k_{0} x \in A_{1} \subseteq W} \bigvee_{\substack{M B \subseteq A_{1} \\
x \in B, k_{0} \in M}} \tau(B) \\
& \leq \bigvee_{\substack{M B \subseteq W \\
k_{0} \in M, x \in B}} \tau(B) \leq \bigvee_{\substack{M B \subseteq W \\
k_{0} \in M}} p_{x}(B) .
\end{aligned}
$$

This completes the proof.

Definition 2.6. Let $(X, \tau)$ and $(Y, \eta)$ be two $L$-fuzzifying topological spaces. A function $f: X \rightarrow Y$ is called an $L$-fuzzifying homeomorphism, if $f$ is a bijection and $f, f^{-1}$ are both continuous.

Proposition 2.7. Let $(X, \tau)$ be an L-fuzzifying topological vector space, $k_{0} \neq 0, k_{0} \in \mathbb{K}$, $x_{0} \in X$. Then the mappings

$$
\begin{gathered}
T_{x_{0}}: X \longrightarrow X, \quad x \longmapsto x+x_{0} ; \\
S_{k_{0}}: X \longrightarrow X, \quad x \longmapsto k_{0} x,
\end{gathered}
$$

are both L-fuzzifying homeomorphisms. 
Proof. Since $T_{x_{0}}^{-1}: X \rightarrow X, x \mapsto x-x_{0}$, it is sufficient to prove that $T_{x_{0}}$ is continuous. For each $x \in X, W \subseteq X$, from $p_{x}(\cdot)$ preserving order and $(X, \tau) L$-fuzzifying topological vector space, we have

$$
\begin{aligned}
p_{x+x_{0}}(W) & \leq \bigvee_{B+C \subseteq W} p_{x}(B) \bigwedge p_{x_{0}}(C) \leq \bigvee_{\substack{B+C \subseteq W \\
x_{0} \in C}} p_{x}(B) \\
& \leq \bigvee_{B+x_{0} \subseteq W} p_{x}(B)=\bigvee_{B \subseteq W-x_{0}} p_{x}(B)=p_{x}\left(W-x_{0}\right)
\end{aligned}
$$

Thus $T_{x_{0}}$ is continuous.

In addition, for fixed $k_{0} \in \mathbb{K}, k_{0} \neq 0$, the inverse of mapping $S_{k_{0}}$ is a function $S_{k_{0}}^{-1}$ : $X \rightarrow X, x \mapsto\left(1 / k_{0}\right) x$. It suffices to prove that $S_{k_{0}}: X \rightarrow X, x \mapsto k_{0} x$ is continuous. For all $x \in X, U \subseteq X$, we have

$$
\begin{aligned}
p_{k_{0} x}(U) & \leq \bigvee_{M V \subseteq U, k_{0} \in M} p_{x}(V) \leq \bigvee_{k_{0} V \subseteq U} p_{x}(V) \\
& =\bigvee_{V \subseteq\left(1 / k_{0}\right) U} p_{x}(V)=p_{x}\left(\left(1 / k_{0}\right) U\right)
\end{aligned}
$$

So the conclusion holds.

Corollary 2.8. Let $(X, \tau)$ be an L-fuzzifying topological vector space and $\theta$ the zero element in $X$. Then for all $x \in X, k \neq 0, k \in \mathbb{K}, A \subseteq X$,

$$
p_{\theta}(A)=p_{x}(x+A), \quad p_{\theta}(A)=p_{\theta}(k A) .
$$

The proof of this corollary is easy.

Corollary 2.9. Let $(X, \tau)$ be an L-fuzzifying topological vector space. Then for all $x \in$ $X, A \subseteq X, k \neq 0, k \in \mathbb{K}$,

$$
\tau(x+A)=\tau(A), \quad \tau(k A)=\tau(A) .
$$

Proof. For each $x \in X, A \subseteq X$, by Corollary 2.8, we have

$$
\begin{aligned}
\tau(x+A) & =\bigwedge_{y \in x+A} p_{y}(x+A)=\bigwedge_{y-x \in A} p_{(y-x)+x}(x+A) \\
& =\bigwedge_{y-x \in A} p_{y-x}(A)=\bigwedge_{z \in A} p_{z}(A)=\tau(A) .
\end{aligned}
$$

The other relation may be obtained by the same method.

Proposition 2.10. Let $(X, \tau)$ be an L-fuzzifying topological vector space and $p_{\theta}(U)>0$. Then for each $x \in X$, there exists $\varepsilon>0$ such that $x \in(1 / \varepsilon) U$.

Proof. For each $x \in X$, since $0 \cdot x=\theta$, it follows from Proposition 2.4 and $U \subseteq X$ that $p_{\theta}(U) \leq \bigvee_{\substack{M V \subseteq U \\ 0 \in M}} p_{x}(V)$. Owing to $p_{\theta}(U)>0$, there exist $0 \in M \subseteq \mathbb{K}, V \subseteq X$ with $M V \subseteq U$ such that $p_{x}(V)>0$. So we have $x \in V$ and $\varepsilon>0$ such that $M=\{k|| k \mid \leq \varepsilon\}$ then $\varepsilon x \in U$.

Thus $x \in(1 / \varepsilon) U$. 


\section{3. $L$-fuzzifying neighborhood structure of zero element in $L$-fuzzifying topological vector spaces}

Theorem 3.1. Let $(X, \tau)$ be an L-fuzzifying topological vector space and $p_{\theta}(\cdot)$ its corresponding L-fuzzifying neighborhood structure of the zero element. Then it has the following properties:

(1) $p_{\theta}(X)=1$;

(2) for all $U \subseteq X, p_{\theta}(U)>0 \Rightarrow \theta \in U$;

(3) for all $U, V \subseteq X, p_{\theta}(U \cap V)=p_{\theta}(U) \wedge p_{\theta}(V)$;

(4) for all $W \subseteq X, p_{\theta}(W) \leq \bigvee_{U+V \subseteq W} p_{\theta}(U) \wedge p_{\theta}(V)$;

(5) for all $U \subseteq X, x \in X, p_{\theta}(U)>0 \Rightarrow$ there exists $\varepsilon>0$ such that $k x \in U$ for all $|k|<\varepsilon$;

(6) for all $U \subseteq X, a \triangleleft p_{\theta}(U)$ implies that there exists a balanced set $V \subseteq U$ such that $a \triangleleft p_{\theta}(V)$.

Conversely, let $X$ be a vector space over $\mathbb{K}$ and let a set-valued function $p(\cdot): 2^{X} \rightarrow L$ satisfy conditions (1), (2), (3), (4), (5), and (6). Then there exists an L-fuzzifying topology $\tau_{p}$ on $X$ such that $\left(X, \tau_{p}\right)$ is an L-fuzzifying topological vector space, and $p(\cdot)$ is an L-fuzzifying neighborhood structure of the zero element.

Proof

Necessity. Conditions (1), (2), and (3) follow directly from the definition of the $L$ fuzzifying neighborhood structure. Condition (5) is obvious by Proposition 2.10. It remains to prove (4) and (6) only.

(4) For all $W \subseteq X$, since the mapping $f$ in Definition 2.1 is continuous and $\theta+\theta=\theta$, we have $p_{\theta}(W) \leq \bigvee_{U+V \subseteq W} p_{\theta}(U) \wedge p_{\theta}(V)$.

(6) For each $U \subseteq X$ and $a \triangleleft p_{\theta}(U)$, from Proposition 2.4, there exist $V_{1} \subseteq X, M \subseteq K$ with $0 \in M$ such that $M V_{1} \subseteq U$ and $a \triangleleft p_{\theta}\left(V_{1}\right)$. Then there is a $\varepsilon>0$ such that $k V_{1} \subseteq U$ for all $|k| \leq \varepsilon$. Put $V=\bigcup_{|k| \leq \varepsilon} k V_{1}$ clearly; $V \subseteq U$ and $\varepsilon V_{1} \subseteq V$. By Corollary 2.8 and $p_{\theta}(\cdot)$ preserving order, we have

$$
p_{\theta}(V) \geq p_{\theta}\left(\varepsilon V_{1}\right)=p_{\theta}\left(V_{1}\right) \triangleright a .
$$

Moreover, we easily find that $V$ is balanced, that is, for each $|k| \leq 1, k V \subseteq V$. This means that the proof of necessity is obtained.

Sufficiency. Suppose that $p(\cdot)$ is a set-valued function from $2^{X} \rightarrow L$ which satisfies conditions (1), (2), (3), (4), (5), and (6). Let $p_{x}(U)=p(U-x)$, for all $x \in X, U \subseteq X$. First we prove that $P=\left\{p_{x} \mid x \in X\right\}$ satisfies conditions (LN1), (LN2), (LN3), and (LN4) in Definition 1.2.

(LN1) For each $x \in X, p_{x}(X)=p(X-x)=p(X)=1$.

(LN2) For each $x \in X$, if $p_{x}(U)>0$, then $p(U-x)>0$. Thus $\theta \in U-x$ from condition (2). So $x \in U$.

(LN3) For all $x \in X, U, V \subseteq X$, we have

$$
\begin{aligned}
p_{x}(U \cap V) & =p(U \cap V-x)=p((U-x) \cap(V-x)) \\
& =p(U-x) \bigwedge p(V-x)=p_{x}(U) \bigwedge p_{x}(V) .
\end{aligned}
$$

(LN4) It remains to prove that condition (u4) in Lemma 1.5 holds. 
First we notice that conditions (3) and (4) imply that

$$
p(U)=\bigvee_{V+V \subseteq U} p(V)
$$

Now we fix an element $x \in X$ and a subset $A$ of $X$. Further, we choose an arbitrary subset $B$ of $X$ and a subset $V$ of $X$ with $V+V \subseteq U=A-x$. Then we distinguish the following cases.

Case 1. $x+V \subseteq B$. Then $p(V)=p_{x}(x+V) \leq p_{x}(B)$.

Case 2. $x+V \nsubseteq B$. Then $(x+V) \cap B^{c} \neq \varnothing$.

Subcase 2.1. For all $y \in(x+V) \cap B^{c}: y+V \subseteq A$,

$$
p(V)=p_{y}(y+V) \leq p_{y}(A), \quad \forall y \in(x+V) \cap B^{c} .
$$

Subcase 2.2. There exists $y \in(x+V) \cap B^{c}$ with $(y+V) \cap A^{c} \neq \varnothing$. Because of $y \in x+V$ we obtain $x+V+V \nsubseteq A$, which is a contradiction to the choice of $V$ ! Hence Case 1 and Case 2.1 only occur. Referring to (3.3) this leads to the estimation

$$
p_{x}(A)=p(A-x)=\bigvee_{V+V \subseteq A-x} p(V) \leq \bigvee_{y \notin B}\left[p_{y}(A) \vee p_{x}(B)\right]
$$

Hence (u4) holds.

By Definition 1.2, there exists an $L$-fuzzifying topology $\tau_{p}$ on $X$ and $\left(p_{x}\right)_{x \in X}$ with $p_{x}(A)=p(A-x)$ a topological $L$-fuzzifying neighborhood structure on $X$ with respect to $\tau_{p}$. Specially, $p(\cdot)$ is an $L$-fuzzifying neighborhood structure of the zero element in $X$.

Then we will prove that the mappings $f$ and $g$ are continuous with respect to $L$ fuzzifying topology $\tau_{p}$.

For each $x, y \in X, W \in 2^{X}$, by condition (4) and $p(\cdot)$ preserving order, we have

$$
\begin{aligned}
p_{x+y}(W) & =p(W-x-y) \leq \bigvee_{U+V \subseteq W-x-y} p(U) \bigwedge p(V) \\
& =\bigvee_{U+V \subseteq W-x-y}\left(\bigvee_{x+B-x \subseteq U} p(B)\right) \bigwedge\left(\bigvee_{y+C-y \subseteq V} p(C)\right) \\
& =\bigvee_{U+V \subseteq W-x-y} \bigvee_{x+B-x \subseteq U} \bigvee_{y+C-y \subseteq V}[p(B) \bigwedge p(C)] \\
& \leq \bigvee_{(x+B)+(y+C) \subseteq W} p(B) \bigwedge p(C)=\bigvee_{B+C \subseteq W}[p(B-x) \bigwedge p(C-y)] \\
& =\bigvee_{B+C \subseteq W} p_{x}(B) \bigwedge p_{y}(C) .
\end{aligned}
$$

So the mapping $f$ in Definition 2.1 is continuous with respect to $\tau_{p}$. 
Finally we prove that the mapping $g$ in Definition 2.1 is continuous with respect to $\tau_{p}$. For each $k_{0} \in \mathbb{K}, x \in X, U \subseteq X$, suppose that $p_{k_{0} x}(U) \neq 0$. Then for each $a \triangleleft p_{k_{0} x}(U)=$ $p\left(U-k_{0} x\right)$, it follows from conditions (4) and (6) that there exists a balanced set $V_{0} \subseteq$ $X$ with $V_{0}+V_{0} \subseteq U-k_{0} x$ such that $a \triangleleft p\left(V_{0}\right)$. By condition (5), we have a $0<t_{0} \in \mathbb{K}$ such that $x \in t V_{0}$, so $\left(k-k_{0}\right) x \in\left(k-k_{0}\right) t_{0} V_{0}$. Denote $s_{0}=\left(1 / t_{0}\right)$; then $\left(k-k_{0}\right) t_{0} V_{0} \subseteq V_{0}$, for all $\left|k-k_{0}\right| \leq s_{0}$. Thus $\left(k-k_{0}\right) x \in V_{0}$, for all $\left|k-k_{0}\right| \leq s_{0}$. For $V_{0} \subseteq X, a \triangleleft p\left(V_{0}\right)$, it follows from (4) that there exists $V_{1} \subseteq X$ with $V_{1}+V_{1} \subseteq V_{0}$ such that $a \triangleleft p\left(V_{1}\right)$. By condition (5) again, we have an $s_{1} \in \mathbb{K}, s_{1} \leq s_{0}$ such that $\left(k-k_{0}\right) x \in V_{1}$, for all $\left|k-k_{0}\right| \leq$ $s_{1}$. Hence $\left(k-k_{0}\right) x+V_{1} \subseteq V_{1}+V_{1} \subseteq V_{0}$. Put $N=\left(\left|k_{0}\right|+1\right)$; from conditions (4) and (6), there exists a balanced set $V_{2} \subseteq X$ with $a \triangleleft p\left(V_{2}\right)$ such that

$$
\underbrace{V_{2}+V_{2}+\cdots+V_{2}}_{N} \subseteq V_{0} \text {. }
$$

Let $s_{2}=N-\left|k_{0}\right|$; then $|k| /\left(\left|k_{0}\right|+s_{2}\right) \leq|k| /\left(\left|k_{0}\right|+\left|k-k_{0}\right|\right) \leq 1$, for all $\left|k-k_{0}\right| \leq s_{2}$. Thus

$$
k V_{2} \subseteq\left(\left|k_{0}\right|+s_{2}\right) V_{2}=\left(\left|k_{0}\right|+1\right) V_{2}=N V_{2} \subseteq V_{0}, \quad \forall\left|k-k_{0}\right| \leq s_{2} .
$$

We denote $s_{3}=\min \left\{s_{1}, s_{2}\right\}$; then for each $k$ with $\left|k-k_{0}\right| \leq s_{3}$, we have $\left(k-k_{0}\right) x+V_{1}+$ $k V_{2} \subseteq V_{0}+V_{0} \subseteq U$. Let $V_{3}=V_{1} \cap V_{2}$; then $a \triangleleft p\left(V_{1}\right) \wedge p\left(V_{2}\right) \leq p\left(V_{3}\right)$. Hence

$$
\begin{aligned}
\left(k-k_{0}\right) x+k V_{3} & \subseteq\left(k-k_{0}\right) x+V_{3}+k V_{3} \subseteq\left(k-k_{0}\right) x+V_{1}+k V_{3} \\
& \subseteq U-k_{0} x, \quad \forall\left|k-k_{0}\right| \leq s_{3} .
\end{aligned}
$$

So for each $k$ with $\left|k-k_{0}\right| \leq s_{3}$, we have $k\left(x+V_{3}\right)=k x+k V_{3} \subseteq U$. Denoting $M=\{k \mid$ $\left.\left|k-k_{0}\right|<s_{3}\right\}$, we have $M\left(x+V_{3}\right) \subseteq U$ and

$$
a \triangleleft p\left(V_{3}\right)=p_{x}\left(x+V_{3}\right) \leq \bigvee_{\substack{M B \subseteq U \\ k_{0} \in M}} p_{x}(B)
$$

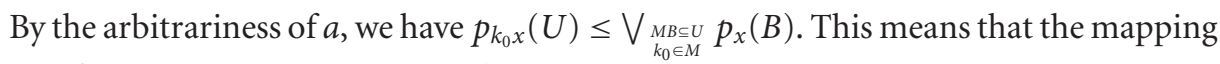
$g$ in Definition 2.1 is continuous with respect to $\tau_{p}$.

Therefore $\left(X, \tau_{p}\right)$ is an $L$-fuzzifying topological vector space, and $p(\cdot)$ is an $L$-fuzzifying neighborhood structure of the zero element.

At the end of this section, we will give a natural class of examples of $L$-fuzzifying topological vector spaces.

Example 3.2. Let $(X, \mathscr{F}, T)$ be a Menger probabilistic normed space [8] with $T=\min$. For each $\varepsilon>0$ and $\lambda \in(0,1], N(\varepsilon, 0)$ and $N(\varepsilon, \lambda)$ are defined as follows:

$$
\begin{gathered}
N(\varepsilon, 0)=\left\{x \in X \mid f_{x}(\varepsilon)=1\right\}, \\
N(\varepsilon, \lambda)=\left\{x \in X \mid f_{x}(\varepsilon)>1-\lambda\right\} .
\end{gathered}
$$


A mapping $p(\cdot): 2^{X} \rightarrow[0,1]$ is defined as follows:

$$
p(A)=\bigvee\{\lambda \mid \exists \varepsilon>0 \text { s.t. } N(\varepsilon, \lambda) \subseteq A\}, \quad \forall A \subseteq X
$$

In what follows we show that the mapping $p(\cdot)$ satisfies conditions (1), (2), (3), (4), (5), and (6) in Theorem 3.1.

It is easy to find that conditions (1), (2), and (6) hold.

(3) By the definition of $p(\cdot)$, it is clear that $p(U \cap V) \leq p(U) \wedge p(V)$, for all $U, V \subseteq$ $X$. Conversely, for each $a \in(0, p(U) \wedge p(V)), a<p(V)$ and $a<p(V)$. Thus there exist $\lambda_{1}, \lambda_{2} \in(0,1], \varepsilon_{1}, \varepsilon_{2}>0$ with $a<\lambda_{1}$ and $a<\lambda_{2}$ such that $N\left(\varepsilon_{1}, \lambda_{1}\right) \subseteq U, N\left(\varepsilon_{2}, \lambda_{2}\right) \subseteq V$. Let $\varepsilon=\varepsilon_{1} \wedge \varepsilon_{2}, \lambda=\lambda_{1} \wedge \lambda_{2}$; then $N(\varepsilon, \lambda)=N\left(\varepsilon_{1}, \lambda_{1}\right) \cap N\left(\varepsilon_{2}, \lambda_{2}\right) \subseteq U \cap V$. So $p(U \cap V) \geq \lambda>$ $a$. By the arbitrariness of $a$, we obtain $p(U) \wedge p(V) \leq p(U \cap V)$. Hence condition (3) holds.

(4) For each $W \subseteq X$, condition (4) holds trivially for the case $p(W)=0$. If $p(W)>0$, then for each $a \in(0, p(W))$, there exist $\varepsilon>0, \lambda \in(0,1]$ with $a<\lambda$ such that $N(\varepsilon, \lambda) \subseteq W$. Taking $\mu \in(a, \lambda)$, we may prove the following relation: $N(\varepsilon / 2, \mu)+N(\varepsilon / 2, \mu) \subseteq N(\varepsilon, \lambda)$.

In fact, since $(X, \mathscr{F}, T)$ is a Menger probabilistic normed space, then for all $x, y \in$ $N(\varepsilon / 2, \mu)$, we have

$$
f_{x+y}(\varepsilon) \geq T\left(f_{x}\left(\frac{\varepsilon}{2}\right), f_{x}\left(\frac{\varepsilon}{2}\right)\right) \geq T(1-\mu, 1-\mu)=1-\mu>1-\lambda
$$

Hence $x+y \in N(\varepsilon, \lambda)$. It follows that $N(\varepsilon / 2, \mu)+N(\varepsilon / 2, \mu) \subseteq N(\varepsilon, \lambda)$.

Let $U=V=N(\varepsilon / 2, \mu)$; then $U+V \subseteq W$, and $p(U) \wedge p(V) \geq \mu>a$. Hence $p(W) \leq$ $\bigvee_{U+V \subseteq W} p(U) \wedge p(V)$.

(5) For each $U \subseteq X$ with $p(U)>0$, there exist $\lambda>0$ and $\varepsilon>0$ such that $N(\varepsilon, \lambda) \subseteq U$. For each $x \in X$, since $\bigvee_{t \geq 0} f_{x}(t)=1>1-\lambda$, there exists $t_{0}>0$ with $f_{x}\left(t_{0}\right)>1-\lambda$. Put $t_{1}=\varepsilon / t_{0}$. Then for all $k \in \mathbb{K}$ with $|k|<t_{1}$, we obtain $f_{k x}(\varepsilon)>1-\lambda$. It follows immediately that $k x \in N(\varepsilon, \lambda) \subseteq U$. Hence condition (5) holds.

By Theorem 3.1, there exists a natural example of $L$-fuzzifying topological vector spaces created by probabilistic normed spaces with respect to $T=\min$.

Remark 3.3. This example was first introduced by Höhle in $[4,5]$. Here we give a direct proof and the definition of $p(\cdot)$ is different from that in $[4,5]$.

\section{Category of $L$-fuzzifying topological vector spaces and category of vector spaces}

In this section, we will use $L$-FYTVS to denote the category of $L$-fuzzifying topological vector spaces, where morphisms are linear continuous mappings.

Theorem 4.1. Let $L$ be a locally multiplicative [12] (this implies that for each $\lambda \in M(L)$, $\lambda \triangleleft b$, and $\lambda \triangleleft c, \lambda \triangleleft b \wedge c$ ) completely distributive lattice and let $U: L-F Y T V S \rightarrow$ VEC be a forgetful functor. Then $U$ is topological. 
Proof. Let $X$ be an object in VEC, $\left(X_{i}, \tau_{i}\right)$ an object in L-FYTVS, and $f_{i}: X \rightarrow U\left(X_{i}\right)$ a morphism in VEC for all $i \in I$. By [1, Definition 21.1 and Definition 21.9], it follows to prove that there exists an $L$-fuzzifying topology $\tau$ on $X$ with $(X, \tau) \in L$-FYTVS such that $\tau$ is the weakest topology with respect to which each mapping $\bar{f}_{i}:(X, \tau) \rightarrow\left(X_{i}, \tau_{i}\right)$ is continuous. Suppose that $p_{i}(\cdot): 2^{X_{i}} \rightarrow L$ is an $L$-fuzzifying neighborhood structure of the zero element in $\left(X_{i}, \tau_{i}\right)$ and $\ll I \gg$ denotes the set of all finite subsets of $I$. Put

$$
p(A)=\bigvee_{V \in \dot{\theta} \mid A} \bigwedge\left\{p_{i}\left(V_{i}\right) \mid \bigcap_{i \in J_{0}} f_{i}^{-1}\left(V_{i}\right)=V, J_{0} \in \ll I \gg\right\}, \quad \forall A \subseteq X
$$

First we prove that the mapping $p(\cdot): 2^{X} \rightarrow L$ satisfies conditions (1), (2), (3), (4), (5), and (6) in Theorem 3.1.

It is easy to find that conditions (1) and (2) hold.

(3) By the definition of $p(\cdot)$, the mapping $p(\cdot)$ preserves order. Then for all $A, B \in 2^{X}$, $p(A \cap B) \leq p(A) \wedge p(B)$. Conversely, for all $a \triangleleft p(A) \wedge p(B)$, we have $a \triangleleft p(A)$ and $a \triangleleft$ $p(B)$. By Lemma 1.6, there exist $V_{1} \in \dot{\theta}\left|A, V_{2} \in \dot{\theta}\right| B$ such that $a \triangleleft \bigwedge\left\{p_{i}\left(A_{i}\right) \mid \bigcap_{i \in J_{0}} f_{i}^{-1}\right.$ $\left.\left(A_{i}\right)=V_{1}, J_{0} \in \ll I \gg\right\}$ and $a \triangleleft \wedge\left\{p_{j}\left(B_{j}\right) \mid \bigcap_{j \in J_{1}} f_{j}^{-1}\left(B_{j}\right)=V_{2}, J_{1} \in \ll I \gg\right\}$. Let $V=$ $V_{1} \cap V_{2}$; it is clear that $V \in \dot{\theta} \mid(A \cap B)$ and $\left(\bigcap_{i \in J_{0}} f_{i}^{-1}\left(A_{i}\right)\right) \cap\left(\bigcap_{j \in J_{1}} f_{j}^{-1}\left(B_{j}\right)\right)=V$. Hence $a \leq p(A \cap B)$. This means that (3) holds.

(4) For each $W \subseteq X$ and every $a \triangleleft p(W)$, there exists $V \in \dot{\theta} \mid W$ such that $a \triangleleft \wedge$ $\left\{p_{i}\left(V_{i}\right) \mid \bigcap_{i \in J_{0}} f_{i}^{-1}\left(V_{i}\right)=V, J_{0} \in \ll I \gg\right\}$. Then $a \triangleleft p_{i}\left(V_{i}\right)$ for all $i \in J_{0}$. Since $\left(X_{i}, \tau_{i}\right) \in L$ FYTVS, there exist $A_{i}, B_{i} \in 2^{X_{i}}$ with $A_{i}+B_{i} \subseteq V_{i}$ such that $a \triangleleft p_{i}\left(A_{i}\right) \wedge p\left(B_{i}\right)$. Put $A=$ $\bigcap_{i \in J_{0}} f_{i}^{-1}\left(A_{i}\right), B=\bigcap_{i \in J_{0}} f_{i}^{-1}\left(B_{i}\right)$; then $A+B \subseteq V$ and $a \leq p(A) \wedge p(B)$. Hence $p(W) \leq$ $\vee_{A+B \subseteq W} p(A) \wedge p(B)$.

(5) For all $A \subseteq X, x \in X$, if $p(A)>0$, then there exists $V \in \dot{\theta} \mid A$ such that $0 \neq \bigwedge\left\{p_{i}\right.$ $\left.\left(V_{i}\right) \mid \bigcap_{i \in J_{0}} f_{i}^{-1}\left(V_{i}\right)=V, J_{0} \in \ll I \gg\right\}$. For each $i \in J_{0}, f_{i}(x) \in X_{i}, p_{i}\left(V_{i}\right)>0$, there is an $\varepsilon_{i}>0$ such that $k f_{i}(x) \in V_{i}$ for all $|k|<\varepsilon_{i}$. Let $\varepsilon=\bigwedge_{i \in J_{0}} \varepsilon_{i}$; clearly $\varepsilon>0$ and $k x \in \bigcap_{i \in J_{0}} f_{i}^{-1}$ $\left(V_{i}\right)=V \subseteq U$ for all $|k|<\varepsilon$.

(6) For each $A \subseteq X$ and every $a \triangleleft p(A)$, there exists $V \in \dot{\theta} \mid A$ such that $a \triangleleft \wedge\left\{p_{i}\left(V_{i}\right) \mid\right.$ $\left.\bigcap_{i \in J_{0}} f_{i}^{-1}\left(V_{i}\right)=V, J_{0} \in \ll I \gg\right\}$. For all $i \in J_{0}$, from $a \triangleleft p_{i}\left(V_{i}\right)$, we have a balanced set $W_{i} \subseteq V_{i}$ such that $a \triangleleft p_{i}\left(W_{i}\right)$. Put $W=\bigcap_{i \in J_{0}} f_{i}^{-1}\left(W_{i}\right)$; it is trivial $W \subseteq U$ and $W$ is a balanced set. Since $L$ is locally multiplicative, we obtain $a \triangleleft \bigwedge_{i \in J_{0}} p_{i}\left(W_{i}\right)$. So $a \triangleleft p(W)$.

Hence there exists an $L$-fuzzifying topology $\tau$ on $X$ such that $(X, \tau) \in L$-FYTVS.

Second we prove that $\tau$ is the weakest $L$-fuzzifying topology such that $\bar{f}_{i}$ is continuous for all $i \in I$.

For each $V_{i} \subseteq X_{i}$ and each $x \in X$,

$$
\begin{aligned}
p_{x}\left(f_{i}^{-1}\left(V_{i}\right)\right) & =p\left(f_{i}^{-1}\left(V_{i}\right)-x\right) \\
& =\bigvee_{A \in \dot{\theta} \mid\left(f_{i}^{-1}\left(V_{i}\right)-x\right)} \bigwedge\left\{p_{j}\left(W_{j}\right) \mid \bigcap_{j \in J_{0}} f_{j}^{-1}\left(W_{j}\right)=A, J_{0} \in \ll I \gg\right\} \\
& \geq p_{i}\left(V_{i}-f_{i}(x)\right)=p_{i, f_{i}(x)}\left(V_{i}\right) .
\end{aligned}
$$

This means that $\bar{f}_{i}:(X, \tau) \rightarrow\left(X_{i}, \tau_{i}\right)$ is continuous. 
In the following we will prove that $\tau$ is coarser than each $L$-fuzzifying topology $\tilde{\tau}$ which makes $\bar{f}_{i}$ continuous for all $i \in I$. In fact, suppose that $\tilde{p}$ is an $L$-fuzzifying neighborhood structure of the zero element with respect to $\tilde{\tau}$. By Lemma 1.3, we prove the next relation $p(A) \leq \tilde{p}(A)$ for all $A \subseteq X$. For each $a \triangleleft p(A)$, there exists $V \in \dot{\theta} \mid A$ such that $a \triangleleft \bigwedge\left\{p_{i}\left(V_{i}\right) \mid \bigcap_{i \in J_{0}} f_{i}^{-1}\left(V_{i}\right)=V, J_{0} \in \ll I \gg\right\}$. Then $a \triangleleft p_{i}\left(V_{i}\right)$ for all $i \in J_{0}$ with $\bigcap_{i \in J_{0}} f_{i}^{-1}\left(V_{i}\right)=V$. By the continuous mapping $\bar{f}_{i}:(X, \tilde{\tau}) \rightarrow\left(X_{i}, \tau_{i}\right)$, we obtain $p_{i}\left(V_{i}\right) \leq$ $\tilde{p}\left(f_{i}^{-1}\left(V_{i}\right)\right)$ for each $i \in J_{0}$. So

$$
a \triangleleft \bigwedge_{i \in J_{0}} \tilde{p}\left(f_{i}^{-1}\left(V_{i}\right)\right)=\tilde{p}\left(\bigcap_{i \in J_{0}} f_{i}^{-1}\left(V_{i}\right)\right)=\tilde{p}(V) \leq \tilde{p}(A)
$$

Hence $\tau \subseteq \tilde{\tau}$. This means that each $U$-structured source $\left(X \rightarrow U\left(X_{i}\right)\right)_{i \in I}$ has a unique $U$-initial lift $\left(X \rightarrow X_{i}\right)_{i \in I}$. This completes the proof.

\section{Acknowledgment}

This project is supported by the Natural Science Foundation of China (no. 10271035) and the Natural Science Foundation of the Educational Committee of Jiangsu Province (no. 04KJB110061).

\section{References}

[1] J. Adámek, H. Herrlich, and G. E. Strecker, Abstract and Concrete Categories, Pure and Applied Mathematics, John Wiley \& Sons, New York, 1990.

[2] J.-X. Fang and C.-H. Yan, L-fuzzy topological vector spaces, J. Fuzzy Math. 5 (1997), no. 1, 133144.

[3] G. Gierz, K. H. Hofmann, K. Keimel, J. D. Lawson, M. W. Mislove, and D. S. Scott, A Compendium of Continuous Lattices, Springer, Berlin, 1980.

[4] U. Höhle, Upper semicontinuous fuzzy sets and applications, J. Math. Anal. Appl. 78 (1980), no. 2, 659-673.

[5] Characterization of L-topologies by L-valued neighborhoods, Mathematics of Fuzzy Sets (S. E. Rodabaugh, E. P. Klement, and U. Hoehle, eds.), Handb. Fuzzy Sets Ser., vol. 3, Kluwer Academic, Massachusetts, 1999, pp. 389-432, Applications of Category Theory to Fuzzy Subsets.

[6] A. K. Katsaras and D. B. Liu, Fuzzy vector spaces and fuzzy topological vector spaces, J. Math. Anal. Appl. 58 (1977), no. 1, 135-146.

[7] Y. Liu and D. Zhang, Lowen spaces, J. Math. Anal. Appl. 241 (2000), no. 1, 30-38.

[8] B. Schweizer and A. Sklar, Probabilistic Metric Spaces, North-Holland Series in Probability and Applied Mathematics, North-Holland, New York, 1983.

[9] M. Ying, A new approach for fuzzy topology. I, Fuzzy Sets and Systems 39 (1991), no. 3, 303321.

[10] A new approach for fuzzy topology. III, Fuzzy Sets and Systems 55 (1993), no. 2, 193207.

[11] G. J. Zhang, Fuzzifying topological linear spaces, Mohu Xitong yu Shuxue 14 (2000), no. 2, 43-47 (Chinese).

[12] D. Zhang, On the reflectivity and coreflectivity of L-fuzzifying topological spaces in L-topological spaces, Acta Math. Sin. (Engl. Ser.) 18 (2002), no. 1, 55-68. 
[13] D. Zhang and L. Xu, Categories isomorphic to FNS, Fuzzy Sets and Systems 104 (1999), no. 3, 373-380.

Cong-hua Yan: Department of Mathematics, Nanjing Normal University, Nanjing, Jiangsu 210097, China

E-mail address: chyan@njnu.edu.cn

Cong-xin Wu: Department of Mathematics, Harbin Institute of Technology, Harbin 150001, China E-mail address: xiaopingxue@263.net 


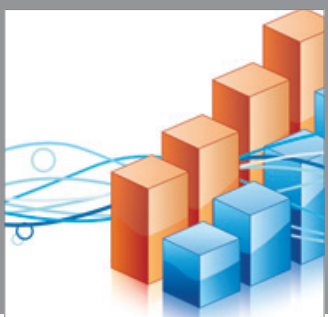

Advances in

Operations Research

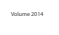

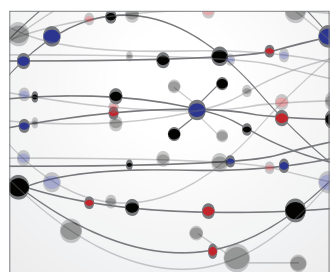

\section{The Scientific} World Journal
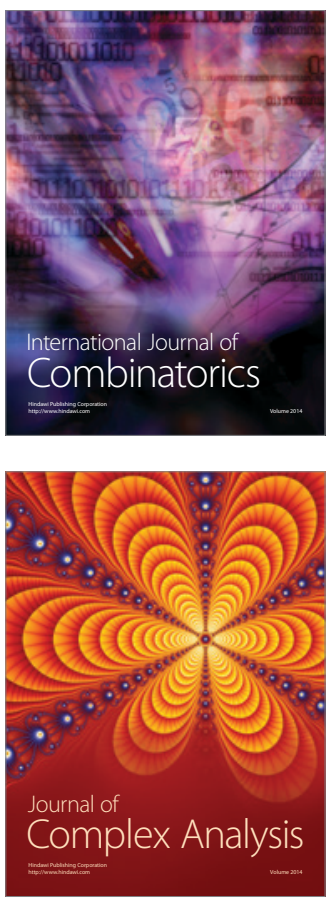

International Journal of

Mathematics and

Mathematical

Sciences
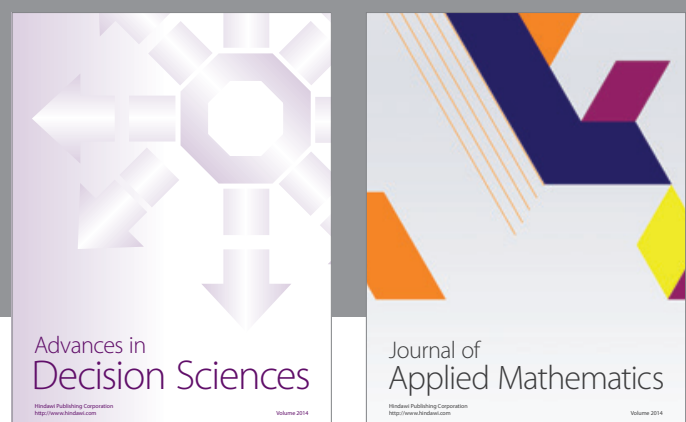

Journal of

Applied Mathematics
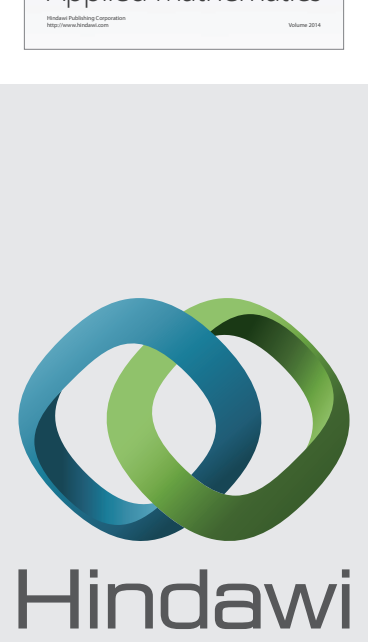

Submit your manuscripts at http://www.hindawi.com
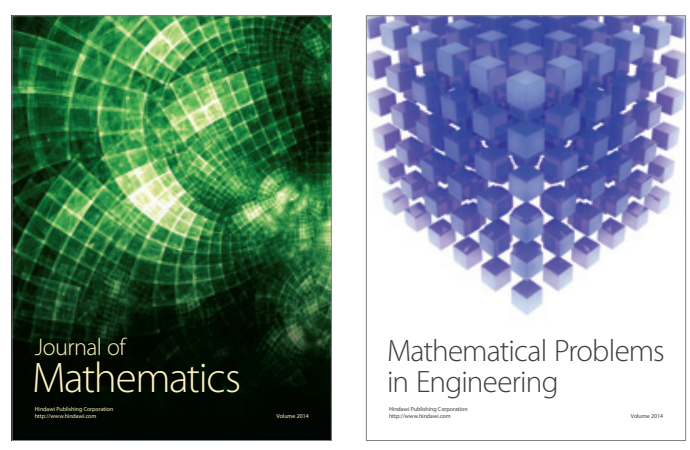

Mathematical Problems in Engineering
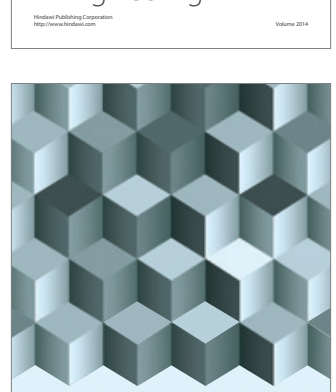

Journal of

Function Spaces
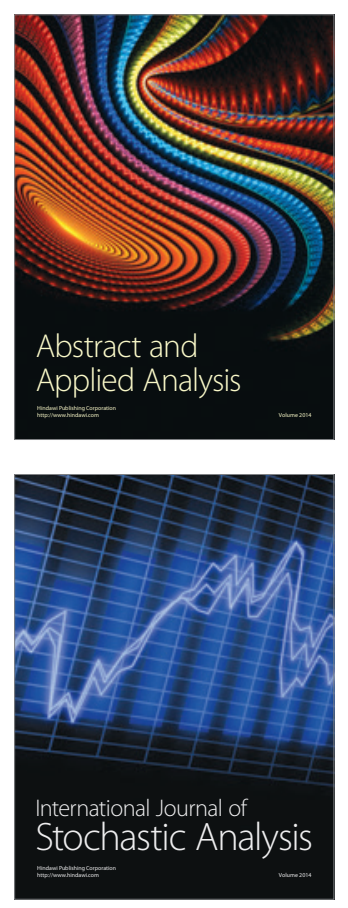

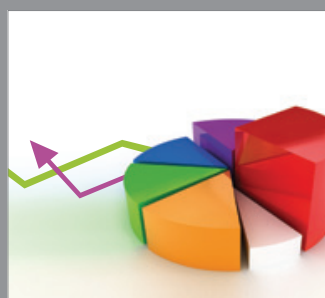

ournal of

Probability and Statistics

Promensencen
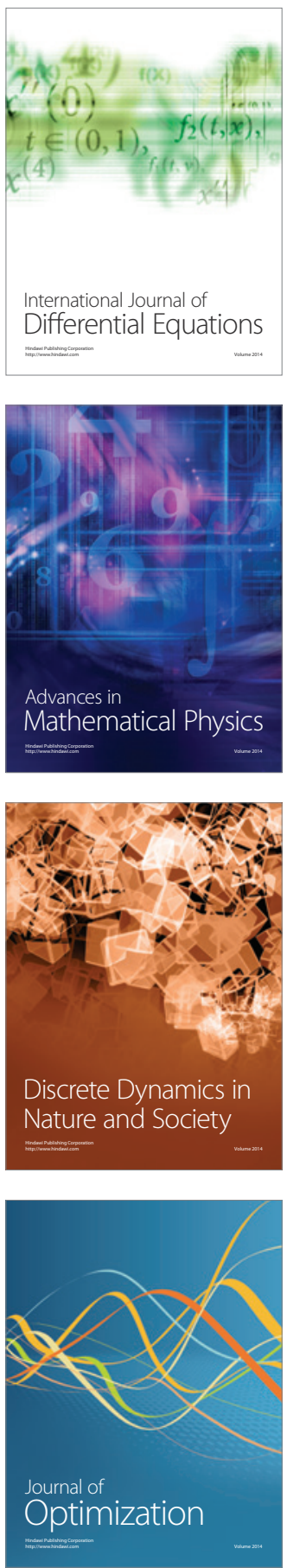